\title{
Actions of Estradiol on Discrete Attributes of the Luteinizing Hormone Pulse Signal in Man

\author{
Studies in Postmenopausal Women Treated with Pure Estradiol
}

\author{
Johannes D. Veldhuis, William S. Evans, Alan D. Rogol, Michael O. Thorner, and Paul Stump; \\ Departments of Internal Medicine, Pharmacology, Pediatrics, and Obstetrics and Gynecology, University of Virginia School of Medicine, \\ Charlottesville, Virginia 22908; *Department of Obstetrics and Gynecology, Jersey Shore Medical Center, Neptune, New Jersey 07753
}

\begin{abstract}
We assessed the time-dependent impact of estradiol on properties of the luteinizing hormone (LH) pulse signal in 12 hypoestrogenemic postmenopausal volunteers studied basally and after 1 , 5,10 , and $30 \mathrm{~d}$ of estradiol delivery via an intravaginal Silastic ring. Computerized analysis of the plasma $\mathrm{LH}$ time series revealed a significant decrease in LH pulse frequency within $24 \mathrm{~h}$ of estrogen treatment, followed by a secondary increase (days 5 and 10), and then a sustained decline (day 30) in LH pulsatility. Estradiol also significantly suppressed incremental and maximal (but not fractional) LH pulse amplitudes in a biphasic manner. In contrast, $\mathbf{L H}$ peak duration was invariant until day 30 of estradiol replacement.

These observations indicate that the well recognized biphasic actions of estradiol on mean serum $\mathrm{LH}$ concentrations can be modeled in relation to specific and time-dependent alterations in LH pulse frequency and amplitude.
\end{abstract}

\section{Introduction}

Investigations of the human menstrual cycle suggest that estradiol can exert both negative feedback (suppressive) and positive feedback (facilitative) effects on gonadotropin secretion (1-5). Moreover, studies in ovariectomized rodents and other mammals subjected to various regimens of sex-steroid hormone replacement have suggested possible actions of estradiol on the hypothalamic release of gonadotropin-releasing hormone (GnRH) ${ }^{1}$ and on the responsiveness of gonadotrope cells to GnRH (613). However, distinct and time-dependent actions of estradiol on the hypothalamic pulse generator and on properties of the endogenous gonadotropin pulse signal have not yet been delineated in man.

To test the specific impact of estradiol on the nature of the endogenous luteinizing hormone (LH) pulse signal, we have employed an intravaginally placed estradiol-impregnated Silastic ring. This mode of estrogen delivery results in sustained increases

Address correspondence to Dr. Johannes Veldhuis, Box 202, Division of Endocrinology and Metabolism, Department of Internal Medicine, University of Virginia School of Medicine, Charlottesville, VA 22908.

Received for publication 23 June 1986 and in revised form $10 \mathrm{No}$ vember 1986.

1. Abbreviations used in this paper: $\mathrm{GnRH}$, gonadotropin-releasing hormone; LH, luteinizing hormone.

J. Clin. Invest.

(c) The American Society for Clinical Investigation, Inc.

0021-9738/87/03/0769/08 \$1.00

Volume 79, March 1987, 769-776 in serum estradiol concentrations without disproportionate rises in plasma estrone levels $(14,15)$. Earlier, we used this model of estradiol administration to elucidate kinetically distinct characteristics of estradiol's effects upon pituitary $\mathrm{LH}$ release in response to exogenous pulses of GnRH in previously hypoestrogenemic postmenopausal women (16). In the present studies, we have extended this approach to investigate discrete and timedependent actions of estradiol on the specific properties of spontaneous pulsatile $\mathrm{LH}$ release.

\section{Methods}

Estradiol administration. Estradiol-containing Silastic rings were prepared exactly as described earlier, with a dose of $400 \mathrm{mg}$ of pure estradiol impregnated within each ring $(14,15)$. Healthy postmenopausal women were studied after provision of written informed consent approved by the Human Investigation Committee of the University of Virginia School of Medicine. The women who participated in this study ranged in age from 55 to $68 \mathrm{yr}(n=12$ women $)$ and were spontaneously postmenopausal by $3.5-10 \mathrm{yr}$ (median, $5 \mathrm{yr}$ ). Each volunteer underwent a detailed history and physical examination (average weight, $56 \pm 2 \mathrm{~kg}$; range, $50-68 \mathrm{~kg}$ ) with documentation of normal hepatic, renal, and hematologic function, biochemical euthyroidism, and postmenopausal concentrations of gonadotropic hormones. At least $6 \mathrm{wk}$ before the study, women were withdrawn from any drugs, including estrogen or other sex-steroid treatments.

Sampling protocols. Sampling was conducted in the Clinical Research Center by withdrawing blood at 10-min intervals for $12 \mathrm{~h}$ (six women) or 20-min intervals for $24 \mathrm{~h}$ (six other women) beginning at 0800 hours. Sampling was performed basally and on days 1, 5, 10, and 30 after initiation of estradiol replacement.

Assays. Serum concentrations of immunoactive $\mathrm{LH}$ were assayed in triplicate exactly as described earlier (17). In this study, the median standard deviations $(\mathrm{mIU} / \mathrm{ml})$ as determined from 96 replicates in this group of 12 women were $2.3,1.7,1.95,1.55$, and 1.45 on days $0,1,5,10$, and 30 of estradiol administration, respectively. Serum concentrations of estradiol were also quantitated by specific radioimmunoassay after chromatography, as published previously (18).

Statistical analysis. Data are expressed as means \pm SEM or median values for the group of 12 volunteers, because the two groups did not differ significantly. Significant overall treatment effects were sought by analysis of variance with the Neuman-Keul's procedure to test for individual significant effects (19). The effects of estrogen on individual specific LH pulse properties were tested within subjects by paired nonparametric methods (Wilcoxon ranked signs test), because the distribution of certain LH pulse properties is non-Gaussian (20). Significance was construed for $P<0.05$.

Pulse analysis. Pulsatile gonadotropin secretion was analyzed by an objective computerized algorithm termed "Cluster analysis" (21). In this procedure, LH series are scanned for all significant increases of a given cluster size (consecutive LH values within a presumptive peak) compared with the preceding "cluster" of nadir LH values. Data are then rescanned in an analogous manner to search for all significant decreases in the series. Significant increases or decreases are judged by pooled $t$ testing using measurement error inherent in the triplicate $\mathrm{LH}$ values in the 

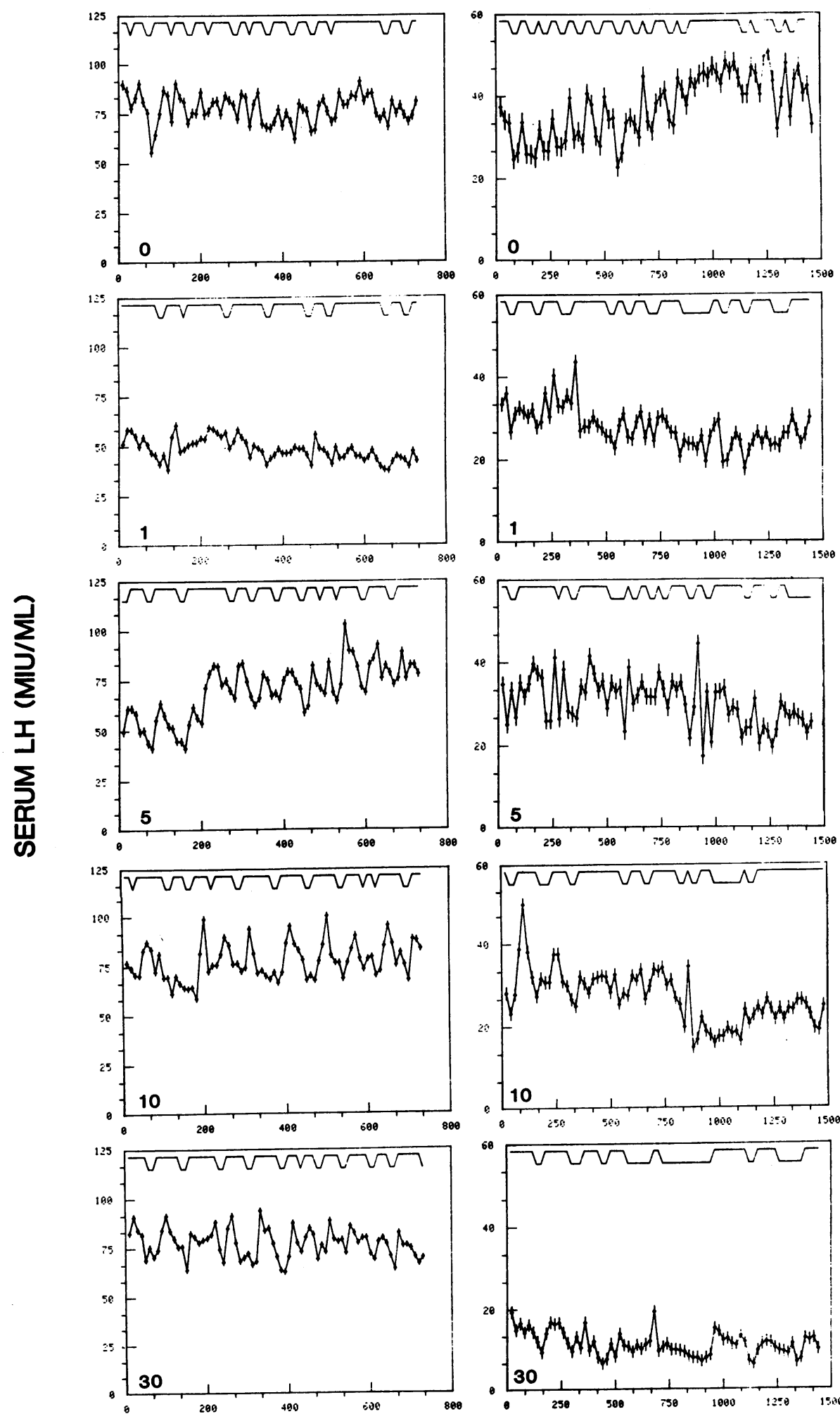

\section{TIME (MIN)}

Figure 1. Time course of LH pulse profiles in two postmenopausal women treated with estradiol for $\mathbf{3 0} \mathrm{d}$. Estradiol was administered via an intravaginally placed Silastic ring impregnated with $400 \mathrm{mg}$ estradiol. Blood sampling was performed every $10 \mathrm{~min}$ for $12 \mathrm{~h}$ (left) or every $\mathbf{2 0} \mathrm{min}$ for $\mathbf{2 4} \mathrm{h}$ (right) on day 0 (basal), and after $1,5,10$, and $30 \mathrm{~d}$ of estradiol replacement. Each subpanel depicts the time course (horizontal axis, min) of the mean \pm SD serum LH concentrations $(\mathrm{mIU} / \mathrm{ml})$ assayed in triplicate for one particular repetitive sampling session (identified in lower left corners as days $0,1,5,10$, or 30 ). The continuous schematized line in the uppermost portion of each panel defines the individually significant LH pulses detected by computer analysis.

cluster. A pulse is defined as a significant increase followed by a significant decrease. Regions of the data series unassociated with either significant increases or significant decreases and flanked by pulses are termed interpulse valleys $(21)$. This program calculates the following parameters: number of significant pulses, interpulse intervals, fractional (percent), incremental, and maximal pulse amplitudes, pulse duration, interpulse valley duration, and valley and nadir mean hormone concentrations. The method has been extensively validated in relation to false-positive 
error rates associated with Gaussian-distributed random measurement error, and appraised in detail in relation to pulsatile $\mathrm{LH}$ release in normal men and women (20).

The Cluster analysis program was applied in the present studies using the following optimized parameters: (a) a $2 \times 1$ cluster configuration (two points in the test nadir, one point in the test peak and two points in the postpeak nadir) in the 20 -min sampling data, and $(b)$ a $2 \times 2$ cluster configuration for the 10 -min sampling data. The respective $t$ statistics chosen were 2.75 for the upstroke and 1.5 for the downstroke (20min sampling), and 2.92 for the upstroke and 1.70 for the downstroke (10-min sampling). These parameters of cluster analysis result in an estimated false-positive rate of $\leq 1.2 \%$ and provide stable estimates of $\mathrm{LH}$ pulse frequency at the respective sampling rates (20).

\section{Results}

Impact of sustained estradiol delivery on mean and integrated serum $L H$ concentrations. As shown in Table I, intravaginal placement of an estradiol-impregnated Silastic ring resulted in a prompt increase in the serum estradiol concentration within $24 \mathrm{~h}$, to a peak value of $325 \pm 34 \mathrm{pg} / \mathrm{ml}$ compared with $11.2 \pm 0.67$ $\mathrm{pg} / \mathrm{ml}$ basally $(P<0.001)$. Serum estradiol concentrations were maintained in the follicular phase range for $30 \mathrm{~d}$, i.e., serum concentrations of $121 \pm 6.9 \mathrm{pg} / \mathrm{ml}$ after $1 \mathrm{mo}$.

Under the above conditions of sustained estradiol delivery, the time-dependent profiles of pulsatile LH release were studied. These profiles are illustrated for two women in Fig. 1. Such extended sampling paradigms (every $10 \mathrm{~min}$ for $12 \mathrm{~h}$ or every $20 \mathrm{~min}$ for $24 \mathrm{~h}$ ) permitted us to calculate accurate mean and integrated serum LH concentrations. Mean serum LH concentrations decreased promptly and significantly from $57 \pm 6.2 \mathrm{mIU} /$ $\mathrm{ml}$ basally (day 0 , estrogen deprived) to $34 \pm 3.1 \mathrm{mIU} / \mathrm{ml}$ within $24 \mathrm{~h}$ of estradiol administration $(P \leq 0.001)$ (Fig. 2). After $5 \mathrm{~d}$ of continued estradiol administration, serum LH concentrations rose to $44 \pm 4.9$ ( $P \leq 0.005$ vs. day 1$)$. This secondary increase in mean serum $\mathrm{LH}$ concentration was followed by a gradual decline on days 10 and $30(P<0.003$ compared with day 0 baseline). In each case, the integrated serum $\mathrm{LH}$ concentrations (integrated over $12 \mathrm{~h}$ in six women and over $24 \mathrm{~h}$ in six other women) exhibited a similar statistically significant biphasic pattern of suppression. The respective areas (given as $\mathrm{IU} / \mathrm{ml} \times 12$ h) were $60 \pm 9.2$ (day 0), $37 \pm 5.1$ (day 1), $49 \pm 8.5$ (day 5), $44 \pm 8.2$ (day 10$)$, and $33 \pm 8.8$ (day 30$)(P=0.001$, overall treatment effect). Thus, the present mode of estradiol replacement provided a model with which to assess the nature of the biphasic actions of estradiol on specific $\mathrm{LH}$ pulse properties.

Table I. Time-dependent Alterations in Mean Serum Estradiol Concentrations in Previously Hypoestrogenemic Postmenopausal Volunteers Receiving Intravaginal Estradiol Via a Silastic Ring

\begin{tabular}{ll}
\hline $\begin{array}{l}\text { Duration of estradiol } \\
\text { replacement }\end{array}$ & Serum estradiol concentration \\
\hline$d$ & $p g / m l$ \\
0 & $11 \pm 0.67^{*}$ \\
1 & $325 \pm 34^{\ddagger}$ \\
5 & $243 \pm 23^{\S}$ \\
10 & $187 \pm 19^{\S}$ \\
30 & $121 \pm 6.9^{\| 1}$
\end{tabular}

Data are means $\pm \operatorname{SEM}$ ( $n=12$ women). Differing superscripts denote significantly different means by analysis of variance.

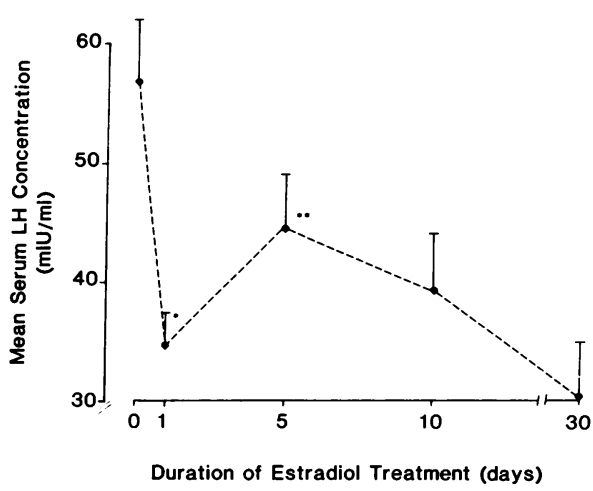

Figure 2. Time-dependent biphasic impact of estradiol replacement on mean serum LH concentrations. Mean serum LH concentrations were determined from 73 blood samples. ${ }^{*} P<0.001$ vs. day $0 .{ }^{* *} P$ $<0.005$ vs. day 1 .

Influence of estradiol on LH interpulse intervals and pulse frequency. The sustained delivery of estradiol via intravaginal Silastic ring to postmenopausal women resulted in a rapid alteration in median LH interpulse intervals (Fig. 3). In particular, the median LH interpulse interval as assessed by Cluster analysis increased from 117 to $144(P=0.013)$ within $24 \mathrm{hrs}$ of estradiol delivery. Thereafter, the $\mathrm{LH}$ interpulse interval declined significantly to a median value of $121 \mathrm{~min}(P=0.020)$ on day 5 . By days 10 and 30 , there was a gradual further prolongation of the median interpulse interval to 130 and $155 \mathrm{~min}$ respectively $(P$ $=0.005$ vs. baseline, day 0 ).

The changes in the measured interpulse interval were mirrored by corresponding changes in LH pulse frequency ( $P$ $=0.008$ for the effect of estradiol on $\mathrm{LH}$ pulse frequency). In particular, estradiol suppressed LH pulse frequency within 24 $\mathrm{h}$ from $7.75 \pm 0.84$ (median 8 ) pulses $/ 12 \mathrm{~h}$ to $6.54 \pm 0.60$ (median 7) pulses/12 h $(P=0.04)$. This immediate suppression of $\mathrm{LH}$ pulse frequency was followed by a secondary increase on day 5 to $7.33 \pm 0.64$ (median 7.5 ) pulses $/ 12 \mathrm{~h}$, and then a decline to below baseline on day 10 (7.14 \pm 0.63 [median 6] pulses/12 h). The lowest LH pulse frequency occurred on day $30: 5.54 \pm 0.68$ (median 4.5$)$ pulses $/ 12$ h $(P=0.008$ vs. basal).

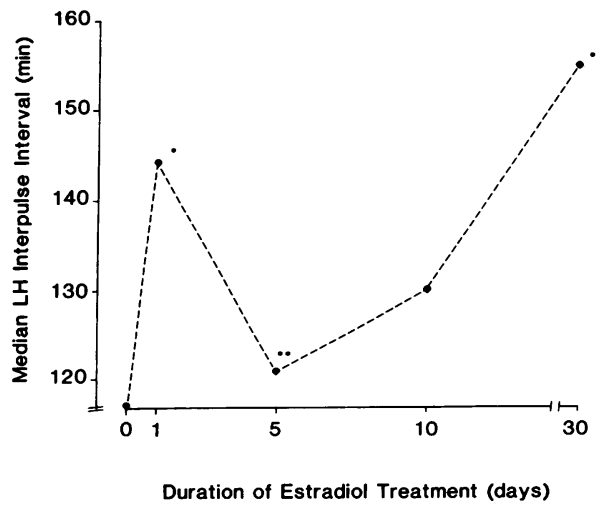

Figure 3. Time-dependent influence of estradiol treatment on the median LH interpulse interval (min). Median $\mathrm{LH}$ interpulse intervals are shown for the group of 12 women replaced with estradiol and sampled at the indicated times during estradiol replacement. ${ }^{*} P \leq 0.005$ vs. day $0 .{ }^{* *} P \leq 0.02$ vs. day 1 . 

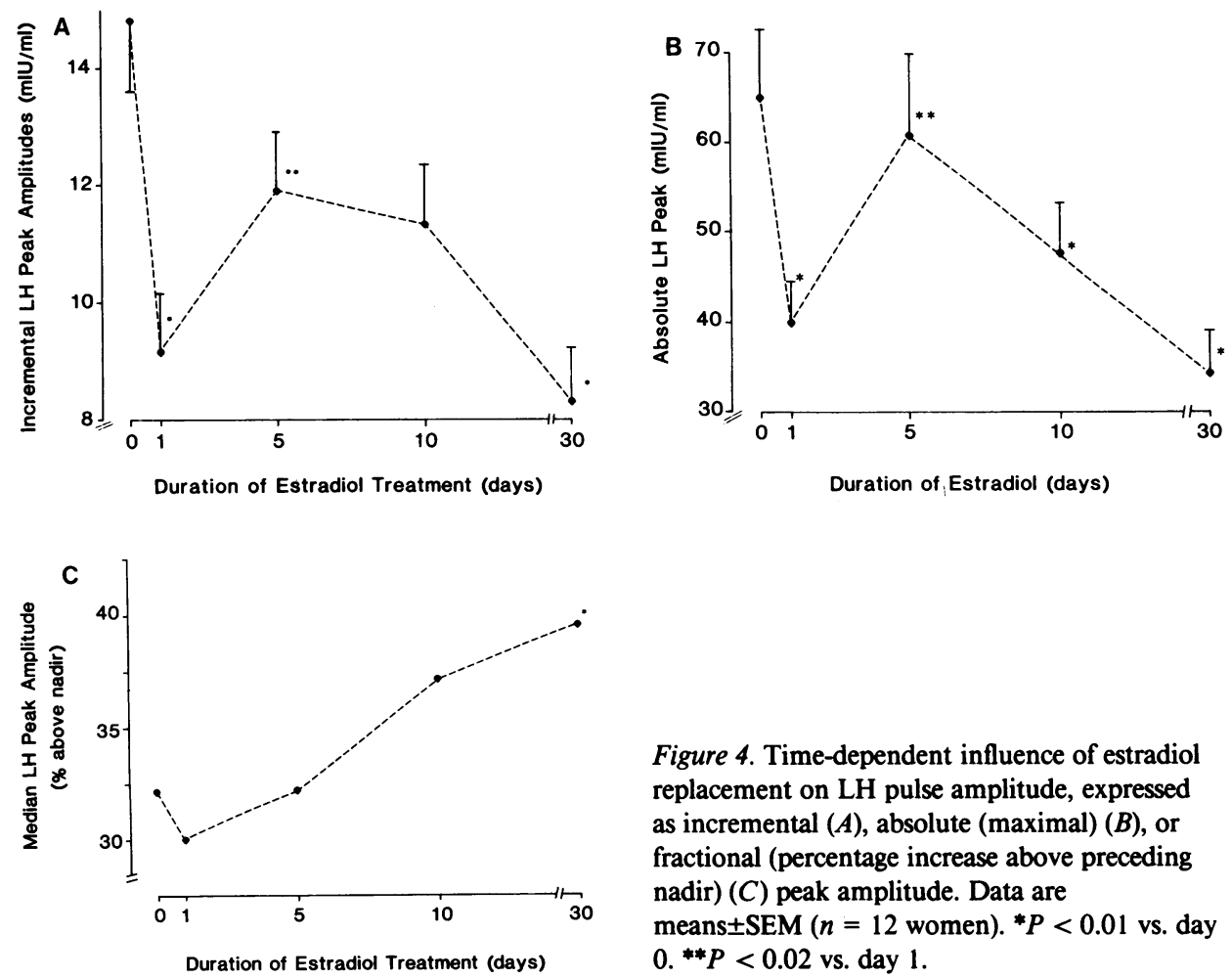

Figure 4. Time-dependent influence of estradiol replacement on LH pulse amplitude, expressed as incremental $(A)$, absolute (maximal) $(B)$, or fractional (percentage increase above preceding nadir) $(C)$ peak amplitude. Data are means $\pm \operatorname{SEM}\left(n=12\right.$ women). ${ }^{*} P<0.01$ vs. day $0 .{ }^{* *} P<0.02$ vs. day 1 .

Time-dependent effects of estradiol replacement on specific $\mathrm{LH}$ pulse properties. The effects of estradiol on specific characteristics of $\mathrm{LH}$ pulse amplitude and duration were examined over the time course of estradiol replacement. We assessed LH pulse amplitude in relation to incremental peak amplitude (mIU/ $\mathrm{ml}$ increment above preceding nadir), absolute LH peak amplitude (maximal LH concentration attained within a peak), and fractional (percentage LH pulse amplitude) increase above preceding nadir. As shown in Fig. 4, $A$ and $B$, incremental and absolute LH peak amplitudes exhibited biphasic responses to sustained estradiol administration with rapid initial suppression, followed by a secondary increase, and then a gradual decline. In contrast, fractional LH pulse amplitude was minimally affected by estradiol administration, except after $30 \mathrm{~d}$ when it increased significantly $(P=0.018)$ (Fig. $4 C)$. The lack of appreciable suppression of $\mathrm{LH}$ fractional pulse amplitude at a time that LH pulse frequency was substantially decreased (day 1) indicates that the lower pulse frequency on day 1 did not represent a false-negative error attributed to a decline in peak amplitude per se.

LH peak duration (minutes) was also altered in a time-dependent manner during estradiol replacement. The changes in LH peak duration in relation to estradiol treatment were somewhat similar to those observed for fractional $\mathrm{LH}$ peak amplitude, in that there was a significant enhancement of peak duration after 30 days of estradiol treatment. Peak duration on day 0 (basal) was 112 \pm 9.3 (median 106) min compared with $141 \pm 14$ (median 124) $\mathrm{min}$ on day $30(P=0.034)$ (Fig. $5 \mathrm{~A})$.

In association with time-dependent changes in incremental LH peak amplitude, there were corresponding significant changes in mean $\mathrm{LH}$ peak area $(\mathrm{mIU} / \mathrm{ml} \times \mathrm{min})($ Fig. $5 \mathrm{~B})$. In particular, mean LH peak area declined significantly within $24 \mathrm{~h}$ of estradiol treatment from $575 \pm 66$ (median 500 ) to $380 \pm 54$ (median 338 ) $(P=0.005$ vs. basal). With continued estrogen exposure, mean LH peak area then increased significantly to $532 \pm 78$ (median 465 ) on day 5 ( $P \leq 0.05$ vs. day 1$)$, before declining again by

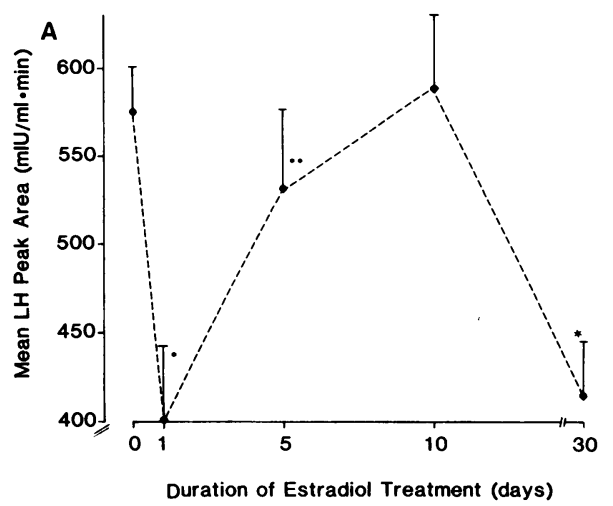

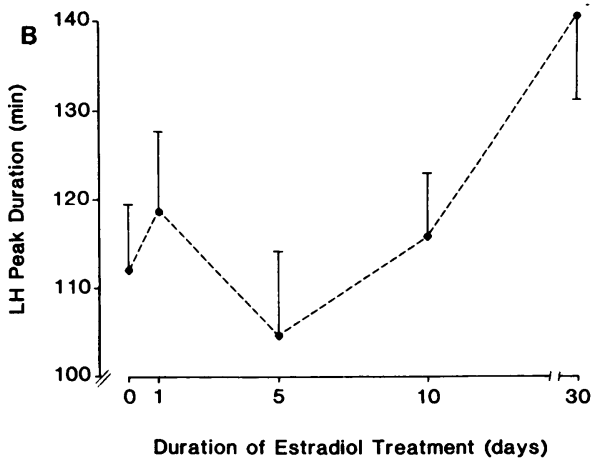

Figure 5. Time-dependent influence of estradiol on mean LH peak area $(A)$ or peak duration $(B)$. Data are means $\pm \operatorname{SEM}\left(n=12\right.$ women). ${ }^{*} P$ $<0.05$ vs. day $0 .{ }^{* *} P<0.05$ vs. day 1 . 


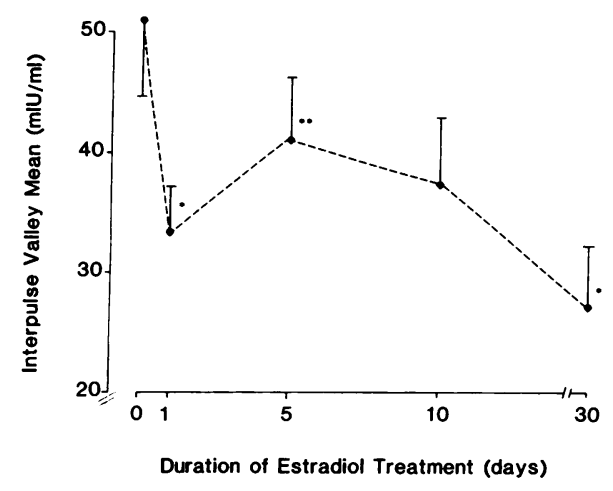

Figure 6. Temporal impact of estradiol replacement on interpulse "valley" mean LH concentrations in 12 previously hypoestrogenemic women. Data are means $\pm \operatorname{SEM}\left(n=12\right.$ women). ${ }^{*} P<0.001$ vs. day $0 .{ }^{* *} P<0.020$ vs. day 1 .

day 30 to a value similar to that observed after $24 \mathrm{~h}$ of estrogen exposure.

Impact of estradiol on interpulse valley mean $\mathrm{LH}$ concentrations. As shown in Fig. 6, interpulse valley mean LH concentrations ( $\mathrm{LH}$ concentrations observed in regions of the $\mathrm{LH}$ series unassociated with any significant increases or decreases in data) also exhibited a biphasic pattern of suppression with significant inhibition on day $1(P=0.003$ vs. basal), followed by a secondary increase on day $5(P=0.014$ vs. day 1$)$, and then a further decline on days 10 and 30 of estradiol replacement (on day 30 , $P=0.003$ vs. basal). The interpulse valley mean $\mathrm{LH}$ concentrations were highly correlated with the corresponding mean $\mathrm{LH}$ concentrations in the 12 women on each of the $5 \mathrm{~d}$ of observation ( $\mathrm{R}=+0.946$ to $+0.993 ; P<0.001$ for each day of observation). Thus, the mean interpulse valley LH concentrations contribute significantly to the overall LH mean.

Correlations among LH pulse properties and serum estradiol concentrations over time. Before estradiol treatment (day 0), mean serum LH concentrations were correlated in a significantly negative fashion with interpulse intervals $(R=-0.668 ; P$ $=0.018$ ). Thus, in the absence of estradiol, high serum LH concentrations were associated with shorter interpulse intervals, i.e., with higher $\mathrm{LH}$ pulse frequencies.

During the period of estradiol's "positive feedback" (days 5 and 10), mean serum LH concentrations were correlated to a significant degree with mean $\mathrm{LH}$ peak areas $(\mathrm{R}=+0.765$ on day 5 ; Fig. $7 \mathrm{~A}$ ). Moreover, after $5 \mathrm{~d}$ of estradiol treatment, mean serum $\mathrm{LH}$ concentrations were significantly positively correlated with serum estradiol in these 12 women $(\mathrm{R}=+0.580 ; P=0.048$; Fig. $7 \mathrm{~B}$ ). Serum estradiol concentrations were also significantly positively correlated with mean $\mathrm{LH}$ peak durations $(\mathrm{R}=+\mathbf{0 . 6 6 4}$; $P=0.024$; day 10) during estrogen's facilitative effects on $\mathbf{L H}$ release (Fig. $7 \mathrm{C}$ ).

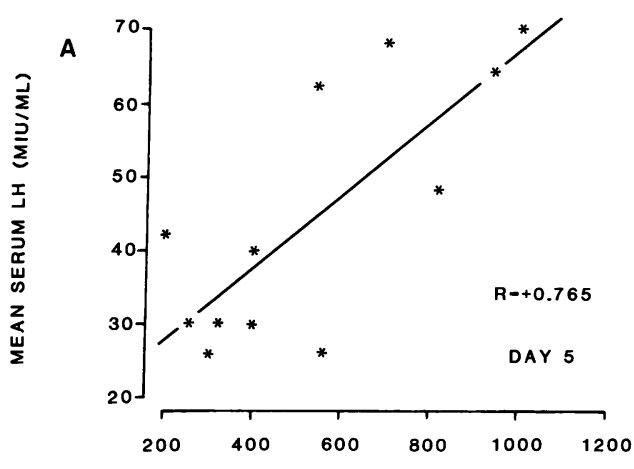

MEAN LH PEAK AREA (MIU/ML $\cdot$ MIN)

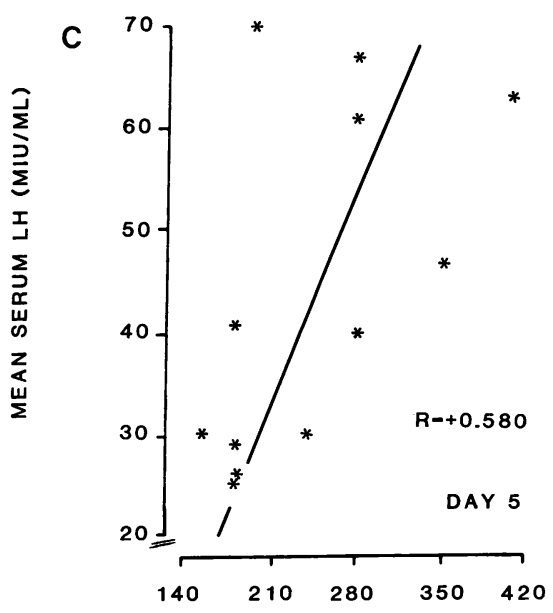

SERUM ESTRADIOL (PG/ML)

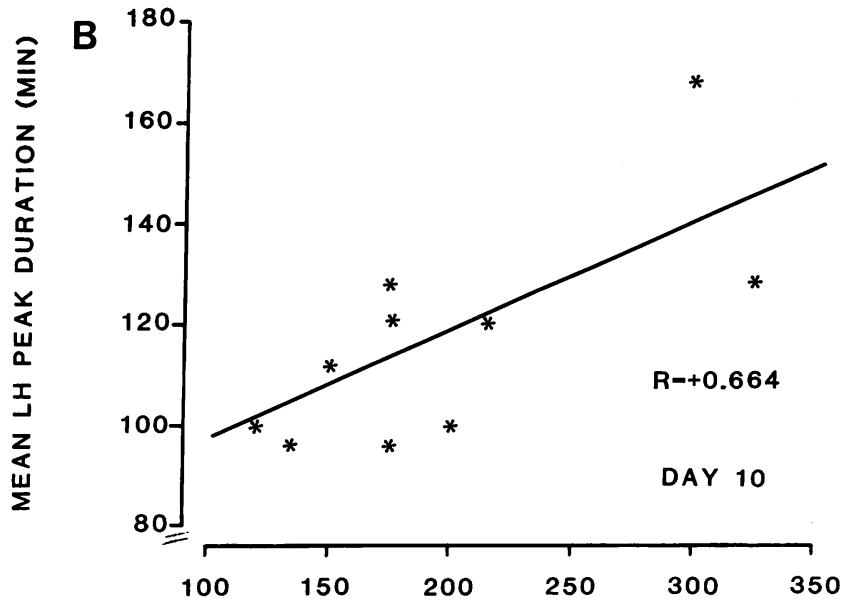

SERUM ESTRADIOL (PG/ML)
Figure 7. Correlations between mean serum $\mathrm{LH}$ concentrations and mean LH peak area and/or mean serum estradiol. Mean serum LH concentrations were significantly positively correlated with mean LH peak areas on day $5(A)$. Mean serum estradiol concentrations were significantly positively correlated with mean LH peak duration on day $10(B)$ and with mean serum LH concentrations on day $5(C)$. 


\section{Discussion}

We have investigated discrete neuroendocrine mechanisms that subserve negative (suppressive) and positive (facilitative) feedback actions of estradiol on LH release in man. We used estradiol-impregnated Silastic rings placed intravaginally to sustain increased circulating estradiol concentrations for $30 \mathrm{~d}$. Whereas this mode of estrogen delivery does not fully recapitulate the temporal profile of endogenous estradiol secretion over the menstrual cycle proper, it can achieve serum estradiol and estrone levels similar to those attained in the mid-to-late follicular phase of the normal menstrual cycle $(14,15)$. Using this model of estradiol administration, we were able to observe a biphasic pattern of gonadotropin release, in which initial suppression of integrated serum LH concentrations (acute negative feedback) was followed by a secondary increase in mean LH concentrations (facilitative or positive feedback effect). This was ultimately superseded by a gradual, continuing decline in mean gonadotropin levels (sustained negative feedback), which were significantly but not completely suppressed. Incomplete suppression of serum LH concentrations at these plasma estradiol concentrations may reflect strength-duration characteristics of estrogen's negative feedback action and/or suggest that other nonestrogenic factors participate in maintaining low circulating levels of $\mathrm{LH}$ in young women with comparable plasma estradiol levels.

The significantly biphasic responses to estradiol observed here mimic in some respects the classical negative and positive feedback actions of estradiol that are believed to be exerted during spontaneous normal menstrual cyclicity and occur in postmenopausal individuals in response to other modes of estrogen replacement $(1-5,22)$. Thus, the present pharmacological model of estrogen action has permitted us to investigate the time-dependent and biphasic impact of estradiol on specific properties of spontaneous pulsatile LH release.

The use of repetitive venous sampling and an objective statistically based pulse detection algorithm has allowed us to unmask significant and temporally constrained actions of estradiol on individual attributes of the LH pulse signal. For example, within $24 \mathrm{~h}$ estradiol administration evoked a significant increase in the $\mathrm{LH}$ interpulse interval in the 12 postmenopausal women studied here. Such an increase in the median LH interpulse interval reflects a significant decline in endogenous LH pulse frequency, rather than a false-negative error in LH peak detection, because fractional LH peak amplitude did not decrease at this time. Thus, the present results indicate that LH pulse frequency is a distinct endpoint of acute estrogen-inhibitory action in man. This acute decline in LH pulse frequency may be of functional significance, because it was paralleled by an immediate decrease in mean circulating $\mathrm{LH}$ concentrations.

The ability of estradiol to elicit a rapid decline in LH pulse frequency presumably reflects a corresponding suppressive effect of estradiol on the frequency of hypothalamic GnRH release, in as much as hypothalamic GnRH release closely mirrors pituitary LH secretion (23). According to this assumption, the present observations would provide evidence for an inhibitory action of estradiol on the hypothalamic regulation of neuroendocrine function in man. Earlier studies using short-term estradiol infusions and less intensive venous sampling in a small number of normal men failed to disclose any effects of injected estradiol on LH pulse frequency $(24,25)$, whereas exogenous estradiol appears to suppress LH pulse frequency in the ovariectomized rat $(10)$, the castrate or anestrous ewe $(9,26)$, and the gonadectomized female rhesus monkey (27). Subsequent efforts in the rodent to localize the anatomic sites of exogenous estradiol's suppressive effects on LH pulse frequency have indicated that only restricted forebrain regions, e.g., the preoptic suprachiasmatic area, are involved (28). Conversely, the use of antiestrogens has provided indirect evidence that endogenous estrogen can tonically suppress LH pulse frequency in man (29-31).

Compared with earlier studies, the present results in menopausal individuals differ not only in experimental context, but also in analytical methods, the schedule of venous sampling, and the number of subjects studied. In particular, we used a validated, statistically based method for peak identification, wherein the false-positive (alpha, or statistical type I) error rate was constrained to $\leq 1.2 \%$ (22). Since the false-positive error rates of earlier analytical methods were not necessarily known or constrained to this particular level, absolute estimates of $\mathrm{LH}$ peak frequency in the literature vary considerably. Absolute estimates of pulse frequency also depend upon the false-negative (beta, or Type II statistical) error rate. Although this error rate in $\mathrm{LH}$ peak detection is not yet known exactly, more intensive and prolonged sampling paradigms are likely to minimize this source of analytical bias. Accordingly, we undertook relatively intensive (blood withdrawal every $10 \mathrm{~min}$ for $12 \mathrm{~h}$ ) and prolonged (blood withdrawal every $20 \mathrm{~min}$ for $24 \mathrm{~h}$ ) sampling protocols. Both of these approaches serve to minimize the counting error inherent in estimating LH pulse frequency (32). In addition, we investigated the effects of estradiol on measured interpulse interval per se, because the latter parameter (unlike pulse number) avoids "edge artifacts" that result when one or more portions of an LH pulse occur at the beginning or end of the sampling session. These technical considerations may have accentuated our ability to delineate for the first time an acute impact of exogenous estradiol on LH pulse frequency in the human.

The decrease in LH pulse frequency observed acutely (within $24 \mathrm{~h}$ ) in response to estradiol administration was temporary, in that after 5 and $10 \mathrm{~d}$ of continued estradiol administration LH pulse frequency increased significantly. This secondary recovery in $\mathrm{LH}$ pulse frequency was associated with a parallel increase in mean and integrated serum $\mathrm{LH}$ concentrations. The latter pattern resembles the so-called positive feedback effect of estradiol observed under physiological and various experimental conditions (1-13). In particular, the present results are consistent with observations in the spontaneously cycling cow, sheep, and human, i.e., estimated LH pulse frequency increases in the late follicular phase as circulating concentrations of estrogenic and other hormones rise before the LH surge $(12,33,34)$. Moreover, in the estrogen-treated ovariectomized rhesus monkey, a burstlike increase in endogenous GnRH neurosecretion occurs in conjunction with the induced LH surge (35). These experimental findings are consistent with our observations on days 5 and 10 of augmented LH pulse frequency in the estrogen-replaced postmenopausal setting.

After inducing a transient increase in $\mathrm{LH}$ pulse frequency (days 5 and 10), continuing estradiol administration for a total of $30 \mathrm{~d}$ resulted in a gradual and significant secondary decrease in LH pulse frequency. Like the acute decline, this delayed decline in LH pulse frequency also could not be attributed to an artifact of the pulse detection algorithm (false-negative error) 
due to low-amplitude LH pulses, because fractional LH pulse amplitude actually increased significantly at this time.

Estradiol administration also evoked significant and timedependent alterations in certain individual properties of $\mathrm{LH}$ pulse amplitude. Thus, estradiol elicited both suppressive and facilitative effects on incremental $(\mathrm{mIU} / \mathrm{ml})$ and absolute (maximal) LH peak amplitudes. The biphasic patterns of these amplitude changes mirrored those of mean and integrated serum LH concentrations, presumably because $\mathrm{LH}$ pulse amplitude reflects in part the magnitude of endogenous LH secretory episodes. In contrast, fractional LH peak amplitude, defined as the percentage increase above preceding nadir, was not significantly influenced by estradiol replacement except on day 30 . This result is consistent with the parallel decline in mean interpulse LH concentrations and incremental LH peak amplitudes. Accordingly, we conclude that the nature of the LH pulse signal, as assessed by its various amplitude attributes, changes significantly and specifically in relation to the time-dependent actions of estradiol.

The present method of analyzing $\mathrm{LH}$ pulse properties quantitatively also permitted us to assess changes in LH peak area and duration, because our statistical algorithm for LH peak detection specifically marks the individually significant upstroke and downstroke bounds of the pulse. We observed that mean LH peak area exhibited a biphasic response to estradiol that mirrored the biphasic pattern of mean serum LH concentrations over time. In contrast, LH peak duration was stable until day 30. Since the relevant physiological characteristic(s) inherent in the LH pulse signal may represent either its frequency, amplitude, and/or duration, the present analyses suggest new mechanisms by which sex steroid feedback could alter the nature, and hence possibly the impact, of the LH pulse signal on the target gonadal cell.

During the time when the presumptive "positive feedback" effects of estradiol occurred (days 5 and 10), serum estradiol concentrations correlated positively with mean plasma LH concentrations and mean LH peak duration (width). In addition, mean LH concentrations correlated positively with endogenous LH peak areas. These observations would be consistent with the ability of estradiol to enhance pituitary responsiveness to exogenous pulses of $\mathrm{GnRH}$ at this time (16), thereby resulting in larger $\mathrm{LH}$ peaks and correspondingly higher integrated $\mathrm{LH}$ concentrations. Such "self-priming" actions of GnRH typically emerge only in an estrogenic milieu, at least when evaluated in response to exogenous pulses of $\mathrm{GnRH}(16,36,37)$. The present study of spontaneous pulsatile LH release indicates that similar correlates of $\mathrm{GnRH}$ self priming in response to estradiol might also occur in response to endogenous $\mathrm{GnRH}$, because these were estrogen-dependent increases in endogenous LH peak amplitude and area. Although increased endogenous LH peak area and/or peak width in the presence of estradiol might reflect alterations in the metabolic clearance rate of $\mathrm{LH}$, available studies during different phases of the normal menstrual cycle indicate that minimal changes occur in LH metabolic clearance despite major changes in endogenous sex steroid hormone concentrations $(38,39)$.

In summary, we have investigated the time-dependent actions of estradiol on specific properties of spontaneous pulsatile LH release in previously hypoestrogenemic postmenopausal women. Sustained parenteral estradiol administration elicited discrete and time-dependent effects on LH pulse frequency, $\mathrm{LH}$ pulse amplitude characteristics, and interpulse mean hormone concentrations. These observations indicate that the negative and positive feedback actions of estradiol, previously recognized in relation to mean serum LH concentrations, are associated with specific alterations in the properties of the endogenous $\mathrm{LH}$ pulse signal.

\section{Acknowledgments}

We thank Chris McNett for her skillful preparation of the manuscript, Paula P. Azimi for the artwork, the National Hormone and Pituitary Program for the provision of purified human LH, E. Elizabeth Taylor and Rebecca Weaver for technical support, Sandra Jackson and the expert nursing staff at the Clinical Research Center at the University of Virginia, and Mr. David Boyd for valued assistance with Clinfo.

This work was supported in part by National Institutes of Health grant RR-00847 to the Clinical Research Center of the University of Virginia, Research Career Development Award No. 1 K04 HD 00634 (JDV), HD 13197 (MOT), HD 19170 (WSE), Diabetes and Research Training Center grant 5 P60 AM 22125-05, and by National Institutes of Health-supported Clinfo Data Reduction Systems.

\section{References}

1. Yen, S. S. C., and C. C. Tsai. 1971. The biphasic pattern in the feedback action of ethinyl estradiol on the release of pituitary FSH and LH. J. Clin. Endocrinol. Metab. 33:882-886.

2. Nillius, S. J., and L. Wide. 1971. Induction of a midcycle-like peak of luteinizing hormone in young women by exogenous oestradiol17ß. J. Obstet. Gynaecol. Br. Commonw. 78:822-829.

3. Yen, S. S. C., and C. C. Tsai. 1972. Acute gonadotropin release induced by exogenous estradiol during the mid-follicular phase of the menstrual cycle. J. Clin. Endocrinol. Metab. 38:298-305.

4. Leyendecker, G., S. Wardlow, and W. Nocke. 1972. Experimental studies on the endocrine regulations during the periovulatory phase of the human menstrual cycle. Acta Endocrinol. 71:160-178.

5. Miyake, A., K. Tasaka, T. Sakumoto, Y. Kawamura, and T. Aono. 1983. Estrogen induces the release of luteinizing hormone-releasing hormone in normal cycling women. J. Clin. Endocrinol. Metab. 56:11001105.

6. Karsch, F. J., R. F. Weick, J. Hotchkiss, D. J. Dierschke, and E. Knobil. 1973. An analysis of the negative feedback control of gonadotropin secretion utilizing chronic implantation of ovarian steroids in ovariectomized rhesus monkeys. Endocrinology. 93:478-486.

7. Goodman, R. I., and F. J. Karsch. 1980. Pulsatile secretion of luteinizing hormone: differential suppression by ovarian steroids. Endocrinology. 107:1286-1290.

8. Noh, K. A., and R. F. Weick. 1981. Effects of oestrogen and progesterone on frequency of pulsatile discharges of luteinizing hormone in the ovariectomized rat. J. Physiol. (Lond.). 319:92P.

9. Martin, G. B., R. J. Scaramuzzi, and J. D. Henstridge. 1983. Effects of oestradiol, progesterone and androstenedione on the pulsatile secretion of luteinizing hormone in ovariectomized ewes during spring and autumn. J. Endocrinol. 96:181-193.

10. Weick, R. F., and K. A. Noh. 1984. Inhibitory effects of estrogen and progesterone on several parameters of pulsatile LH release in the ovariectomized rat. Neuroendocrinology. 38:351-356.

11. Weick, R. F., V. Pitelka, and D. L. Thompson. 1983. Separate negative feedback effects of estrogen on the pituitary and the central nervous system in the ovariectomized rhesus monkey. Endocrinology. 112:1862-1864.

12. Karsch, F. J., D. L. Foster, E. L. Bittman, and R. L. Goodman. 1983. A role for estradiol in enhancing luteinizing hormone pulse frequency during the follicular phase of the estrous cycle of sheep. Endocrinology. 113:1333-1338.

13. Asch, R. H., J. P. Balmaceda, M. R. Borghi, R. Niesvisky, D. H. Coy, and A. V. Schally. 1983. Suppression of positive feedback of estradiol 
benzoate on gonadotropin secretion by an inhibitory analog of luteinizing hormone-releasing hormone (LRH) in oophorectomized rhesus monkeys: evidence for a necessary synergism between LRH and estrogens. J. Clin. Endocrinol. Metab. 57:367-372.

14. Stumpf, P. G., J. Maruca, R. J. Santen, and L. M. Demers. 1982. Development of a vaginal ring for achieving physiologic levels of $17 \beta$ estradiol in hypoestrogenic women. J. Clin. Endocrinol. Metab. 54:208211.

15. Veldhuis, J. D., E. Samojlik, W. S. Evans, A. D. Rogol, C. E. Ridgeway, W. F. Crowley, L. Kolp, E. Checinska, M. A. Kirscher, M. O. Thorner, and P. Stumpf. 1986. Endocrine impact of pure estradiol replacement in postmenopausal women: alterations in anterior pituitary hormone release and circulating sex steroid hormone concentrations. Am. J. Obstet. Gynecol. 155:334-340.

16. Veldhuis, J. D., W. S. Evans, A. D. Rogol, L. Kolp, M. O. Thorner, and P. Stumpf. 1986. The pituitary self-priming actions of gonadotropinreleasing hormone: kinetics of estradiol's potentiating effects on GnRHfacilitated $\mathrm{LH}$ and FSH release in healthy post-menopausal women. $J$. Clin. Invest. 77:1849-1856.

17. Veldhuis, J. D., W. S. Evans, A. D. Rogol, M. O. Thorner, C. R. Drake, G. R. Merriam, and M. L. Johnson. 1984. Intensified rates of venous sampling unmask the presence of spontaneous high-frequency pulsations of luteinizing hormone in man. J. Clin. Endocrinol. Metab. 59:96-102.

18. Samojlik, E., J. D. Veldhuis, S. A. Wells, and R. J. Santen. 1980. Preservation of androgen secretion during estrogen suppression with aminoglutethimide in the treatment of metastatic breast carcinoma. $J$. Clin. Invest. 65:602-612.

19. Winer, B. J. 1971. Statistical Principals in Experimental Design. McGraw-Hill Book Co., New York. p. 196.

20. Urban, R. J., A. D. Rogol, W. S. Evans, M. L. Johnson, and J. D. Veldhuis. 1987. Contemporary aspects of endocrine signal analysis: the paradigm of the luteinizing hormone pulse signal in man. Endocrine Reviews. In press.

21. Veldhuis, J. D., and M. L. Johnson. 1986. Cluster analysis: a simple, versatile and robust algorithm for endocrine pulse detection. Am. J. Physiol. 250:E486-E493.

22. Keye, W. R., Jr., and R. B. Jaffe. 1975. Strength-duration characteristics of estrogen effects on gonadotropin response to gonadotropinreleasing hormone in women. I. Effects of varying duration of estradiol administration. J. Clin. Endocrinol. Metab. 41:1003-1008.

23. Clarke, I. J., and J. T. Cummins. Increased gonadotropin-releasing hormone pulse frequency associated with estrogen-induced luteinizing hormone surges in ovariectomized ewes. Endocrinology. 116:2376-2379.

24. Santen, R. J. 1975. Is aromatization of testosterone to estradiol required for inhibition of luteinizing hormone secretion in men. J. Clin. Invest. 56:1555-1563.

25. Lasley, B. L., C. F. Wang, and S. S. C. Yen. 1976. Assessment of the functional capacity of the gonadotrophs in men: effects of estrogen and clomiphene. J. Clin. Endocrinol. Metab. 43:182-189.

26. Goodman, R. L., E. L. Bittman, D. L. Foster, and F. J. Karsch.
1982. Alterations in the control of luteinizing hormone pulse frequency underlie the seasonal variation in estradiol negative feedback in the ewe. Biol. Reprod. 27:580-589.

27. Yamaji, T., D. J. Dierschke, N. Bhattacharya, and E. Knobil. 1972. The negative feedback control by estradiol and progesterone of LH secretion in the ovariectomized rhesus monkey. Endocrinology. 90: 771-777.

28. Akema, T., Y. Tadokoro, and F. Kimura. 1984. Regional specificity in the effect of estrogen implantation within the forebrain on the frequency of pulsatile luteinizing hormone secretion in the ovariectomized rat. Neuroendocrinology. 39:517-523.

29. Santen, R. J., and E. B. Ruby. 1979. Enhanced frequency and magnitude of episodic luteinizing-hormone releasing hormone discharge as a hypothalamic mechanism for increased luteinizing hormone secretion. J. Clin. Endocrinol. Metab. 48:315-319.

30. Winters, S. J., and P. Troen. 1985. Evidence for a role of endogenous estrogen in the hypothalamic control of gonadotropin secretion in man. J. Clin. Endocrinol. Metab. 61:842-845.

31. Kerin, J. F., J. H. Lin, G. Phillipou, and S. S. C. Yen. 1985. Evidence for a hypothalamic site of action of clomiphene citrate in women. J. Clin. Endocrinol. Metab. 61:265-268.

32. Veldhuis, J. D., W. S. Evans, M. L. Johnson, and A. D. Rogol. 1986. Physiological properties of the luteinizing hormone pulse signal: impact of intensive and extended venous sampling paradigms on their characterization in healthy men and women. J. Clin. Endocrinol. Metab. 62:881-891.

33. Rahe, C. H., R. E. Owens, J. L. Fleeger, H. J. Newton, and P. G. Harms. 1980. Pattern of plasma luteinizing hormone in the cyclic cow: dependence upon the period of the cycle. Endocrinology. 107:498-503.

34. Yen, S. S. C., C. C. Tsai, F. Naftolin, G. Vandenberg, and L. Ajabor. 1972. Pulsatile patterns of gonadotropin release in subjects with and without ovarian function. J. Clin. Endocrinol. Metab. 34:671-674.

35. Levine, J. E., R. L. Norman, P. M. Gleissman, T. T. Oyama, D. R. Bangsberg, and H. G. Spies. 1985. In vivo gonadotropin-releasing hormone release and serum luteinizing hormone measurements in ovariectomized, estrogen-treated rhesus macaques. Endocrinology. 117: 711-721.

36. Yen, S. S. C., G. VandenBerg, and T. M. Siler. 1974. Modulation of pituitary responsiveness to LRF by estrogen. J. Clin. Endocrinol. Metab. 39:170-177.

37. Wang, C. F., B. L. Lasley, A. Lein, and S. S. C. Yen. 1976. The functional changes of pituitary gonadotrophs during the menstrual cycle. J. Clin. Endocrinol. Metab. 42:718-728.

38. Weick, R. F. 1977. A comparison of the disappearance rates of luteinizing hormone from intact and ovariectomized rats. Endocrinology. 101:157-161.

39. Wehmann, R. E., M. R. Blackman, and S. M. Harman. 1982. Metabolic clearance rates of luteinizing hormone in women during different phases of the menstrual cycle and while taking an oral contraceptive. J. Clin. Endocrinol. Metab. 55:654-659. 